\title{
Expression of NUCB2/nesfatin-1 in the taste buds of rats
}

\author{
Xun $\mathrm{Cao}^{1)}$, Xiao Zhou $^{2)}$, Yang $\mathrm{Cao}^{1)}$, Xiao-Min Liu ${ }^{1)}$ and Li-Hong Zhou ${ }^{1)}$ \\ 1) Department of Endocrinology, Harbin Medical University, Harbin 150001, Heilongjiang Province, China \\ 2) Department of Human Anatomy and Embryology, Harbin Medical University, Harbin 150001, Heilongjiang Province, China
}

\begin{abstract}
Nesfatin-1, an anorexigenic peptide derived from nucleobindin 2 (NUCB2), is closely involved in feeding behavior, glycometabolism, and satiety regulation. Some studies show that NUCB2/nesfatin-1 is highly expressed and interacts with many appetite-regulating peptides that are co-expressed in the gastrointestinal tract. However, it remains unclear whether nesfatin-1 is expressed and interacts similarly in taste buds. Glucagon-like peptide-1 (GLP-1), a wellknown appetite down-regulating peptide, is associated with changes in the expression of nesfatin-1. Therefore, we measured the expression of the NUCB2 gene and the distribution of nesfatin-1-immunoreactive cells and investigated whether these variables change in taste buds of circumvallate papillae (CV) from rats with type 2 diabetes (T2DM) after treatment with liraglutide, a GLP-1 receptor agonist. The results showed that nesfatin-1 immunoreactive cells were localized in the taste buds of rat CV. Quantitative RT-PCR showed a significantly lower expression of NUCB2 mRNA in the taste buds of diabetic control rats (T2DM-C) than in those of the normal control group (NC) and a higher level of NUCB2 in the liraglutide treated group (T2DM + LIR) than either the T2DM-C or the NC groups. Changes in the expression of NUCB2 in the rat hypothalamus were opposite to those in CV taste buds. In summary, we found that rat $\mathrm{CV}$ taste buds express NUCB2/nesfatin-1, and that this expression decreases significantly in T2DM and increases after treatment with liraglutide in rat CV. This indicates that nesfatin-1 could be an important factor in the regulation of gustatory function, feeding and perhaps energy homeostasis.
\end{abstract}

Key words: Nesfatin-1, Taste, T2DM, Liraglutide

NESFATIN-1 is an 82-amino-acid peptide derived from posttranslational processing of the $\mathrm{N}$-terminal fragment of nucleobindin 2 (NUCB2), which was identified originally as an anorexigenic hypothalamic neuropeptide [1]. This peptide exhibits many functions in different tissues, especially on feeding and glycometabolism. Nesfatin-1 can decrease food intake and body weight, delay gastric emptying, and increase glucose-induced insulin secretion and glucose elimination [2-4]. Nesfatin-1 can also pass through the blood-brain barrier without saturation, suggesting a peripheral source [5]. Either peripheral or central administration of nesfatin- 1 can reduce food intake through a leptinindependent mechanism [6]. In addition to the hypo-

Submitted Aug. 22, 2015; Accepted Sep. 24, 2015 as EJ15-0489 Released online in J-STAGE as advance publication Oct. 30, 2015 Correspondence to: Xiao-Min Liu, Department of Endocrinology, Harbin Medical University, No. 23 Youzheng street, Harbin 150001, Heilongjiang Province, China. E-mail: liuxiaomin57@aliyun.com Correspondence to: Li-Hong Zhou, Department of Endocrinology, Harbin Medical University, No. 23 Youzheng street, Harbin 150001, Heilongjiang Province, China. E-mail: zlh1972@126.com thalamus, NUCB2/nesfatin-1 is broadly expressed in peripheral tissues including the gastrointestinal tract $[7,8]$. However, there are no reports about whether NUCB2/nesfatin-1 is expressed in rat taste buds.

Recently, a number of reports have shown that many gastrointestinal acting peptides, such as cholecystokinin (CCK), glucagon-like peptide-1 (GLP-1), and ghrelin that are classically considered to regulate appetite, also exist in taste cells and may play a role in the gustatory system [9-12]. Of these peptides, ghrelin is the only known hormone originating from gastric X/Alike cells that can stimulate food intake centrally [13]. Interestingly, ghrelin and nesfatin-1 are co-localized in gastric X/A-like cells, but they antagonize each other [7].

As a member of the incretin family, GLP-1 is released from intestinal L-cells in response to dietary nutrient intake $[14,15]$. Available data suggest that GLP-1 has many beneficial endocrine and metabolic effects including reduction of appetite and body weight, delay of gastric emptying, and decrease of blood sugar, mainly by promoting glucose-dependent insulin secretion $[16,17]$. 
Impairment of taste is a complication of diabetes mellitus [18]. Chronic hyperglycemia can decrease an individual's ability to detect and recognize sweet, salty, and bitter solutions [19]. Taste disturbances like ageusia (taste loss), hypogeusia (decrease in taste), and dysgeusia (abnormal taste) are important and easily neglected manifestations of diabetes [20].

Liraglutide has $97 \%$ sequence identity to native GLP-1 and contains an additional 16-carbon fatty acid that can serve as a long-acting GLP-1 receptor (GLP-1R) agonist. It is used for the treatment of type 2 diabetes mellitus (T2DM) and obesity [21], although the exact mechanisms by which liraglutide induces weight loss are not fully understood. Recently, we and other groups have separately found that GLP-1 and GLP-1R are expressed in the taste buds of both animals and humans, that GLP-1 signaling acts to enhance sweet taste sensitivity $[10,22]$, and that there are significant changes in these parameters in diabetic and/ or obese individual. This indicates that GLP-1 is an important peptide for the regulation of taste senses $[23,24]$. Ramesh N et al. [25] recently reported that NUCB2/Nesfatin-1 was co-localized with incretins GLP-1 and glucose dependent insulinotropic polypeptide (GIP) in mouse small intestinal cells and it stimulates both GLP-1 and GIP mRNA expression and secretion. However, there are few studies regarding the relationship between GLP-1 and NUCB2/nesfatin-1 in taste buds or the hypothalamus. Therefore, the current study was designed to explore whether nesfatin-1 is co-expressed with other appetites-regulating peptides in rat taste buds, and whether there are any changes in their relationship in diabetic and/or obese rats, especially after treatment with liraglutide.

\section{Materials and Methods}

\footnotetext{
Animals

Forty two male Wistar rats (purchased from Liaoning ChangSheng Biotechnology Co., Liaoning, China), weighing 180-200g, 8-10 weeks old, were individually housed under controlled room temperature $\left(22 \pm 2^{\circ} \mathrm{C}\right)$ and humidity ( $55 \pm 5 \%$ ), with a $12: 12 \mathrm{~h}$ light-dark cycle. Water and food were available ad libitum. All procedures adhered to the National Institutes of Health Guide for the Care and Use of Laboratory Animals. After a 1-week habituation, rats were randomly divided into high-fat $(\mathrm{n}=30$, diet contained $45 \%$ carbohydate, $15 \%$ protein, $40 \%$ fat) and normal control (NC, $\mathrm{n}=$
}

12 , diet contained $68 \%$ carbohydate, $23 \%$ protein, $9 \%$ fat) diet groups. After 8 weeks, the rats fed a highfat diet were treated by intraperitoneal injection of 30 $\mathrm{mg} / \mathrm{kg}$ streptozotocin (Sigma-Aldrich, St. Louis, MO, USA), while NC rats received a comparable injection of saline. Seventy-two hours following the treatment, 24 rats with non-fasting blood glucose levels of $\geq 300$ $\mathrm{mg} / \mathrm{dL}(16.7 \mathrm{mM})$ were considered T2DM rats [26] and continued on the high fat diet. Eight weeks later, 12 of these rats were treated daily with liraglutide (T2DM + LIR, $0.2 \mathrm{mg} / \mathrm{kg}$, s.c.) for 4 weeks, the remaining 12 diabetic rats served as the diabetic control (T2DM-C) group. Both NC and T2DM-C rats were injected with saline once daily. Food intake was recorded twice a week and body weights (BWs) and non-fasting blood glucose (BG) levels were determined weekly.

\section{Blood assays}

After 4 weeks of treatment with liraglutide, fasted rats were injected intraperitoneally with $10 \%$ chloral hydrate $(0.3 \mathrm{~mL} / 100 \mathrm{~g})$ after overnight food deprivation. BWs were measured and blood was drawn from the right ventricle. BG was assayed immediately by using the hexokinase method. Serum samples were stored at $-80^{\circ} \mathrm{C}$ until assayed. Blood total triglyceride (TG) and cholesterol (TC) were measured by commercial assay kits (Huili Biotech, Changchun, China). Insulin (FINS) was quantified by an ELISA kit (EMD Millipore, Billerica, MA, USA).

\section{Immunohistochemistry}

Small blocks containing the circumvallate papillae (CV) of rats were quickly dissected immediately after euthanasia. The streptavidin-peroxidase (SP) method was used for immunohistochemical staining. Excised tissues were immersed in a solution of $4 \%$ paraformaldehyde for $24 \mathrm{~h}$ and processed for embedding in paraffin wax. The $7 \mu \mathrm{m}$-thick sections were cut serially. Tissue sections were de-waxed and rehydrated, rinsed with distilled water and phosphate-buffered saline (PBS), quenched with $3 \% \mathrm{H}_{2} \mathrm{O}_{2}$, and incubated with citrate buffer ( $\mathrm{pH} \mathrm{6.0)}$ ) for $20 \mathrm{~min}$ at $100^{\circ} \mathrm{C}$ for antigen retrieval [27]. Then non-specific binding was reduced by pretreatment with 3\% normal goat serum for 20 min and incubated with a rabbit polyclonal antibody against NUCB2/Nesfatin-1 (Phoenix Pharmaceuticals, Burlingame, CA, USA) at a dilution of $1: 800$ at $4{ }^{\circ} \mathrm{C}$ overnight. In addition, in order to examine staining using adjacent slices, two adjacent slices were 
incubated separately with the primary antibody antiNUCB2/Nesfatin-1 and anti- $\alpha$-gustducin (at a dilution of 1:200, Santa Cruz Biotechnology Inc., Santa Cruz, CA, USA). Slides were then washed with PBS. The secondary goat anti-rabbit IgG antibody (Boster, Wuhan, China) was applied at room temperature followed by a PBS rinse. Sections were developed by reaction in $0.05 \% 3,3^{\prime}$-diaminobenzidine tetrahydrochloride and $\mathrm{H}_{2} \mathrm{O}_{2}$. Sections were then rinsed, dehydrated through a graded series of ethanol, cleared in xylene, and cover-slipped.

Slides were observed under light microscopy. Each slide was examined by two independent observers. Twenty immunopositive taste buds from each rat were randomly selected to count nesfatin-1-immunopositive cells. Data were analyzed quantitatively by cell counts according to the protocol described previously [28].

\section{Total RNA extraction and quantitative RT-PCR (RT-qPCR)}

$\mathrm{CV}$ taste buds and hypothalami were collected quickly and stored at $-80^{\circ} \mathrm{C}$. Total RNA was extracted using the Trizol reagent according to the manufacturer's protocol (Roche Applied Science, Mannheim, Germany). After assaying the RNA concentration using a Multiskan Spectrum spectrophotometer (Thermo Fisher Scientific, Vantaa, Finland), RNA was reverse-transcribed to cDNA with a commercial PrimeScript RT Reagent Kit (Takara Biotechnology, Dalian, China). The resulting materials were amplified by PCR using gene-specific primer pairs (Table 1) and the SYBR Green PCR Master Mix (Roche Applied Science, Mannheim, Germany).

For RT-qPCR, amplification conditions were $95^{\circ} \mathrm{C}$ (10min), and then 40 cycles at $95^{\circ} \mathrm{C}(15 \mathrm{sec})$ and $60^{\circ} \mathrm{C}$ (1min). $\beta$-Actin was used as an internal control to normalize the amount of input RNA. Relative mRNA expression levels were calculated by the cycle threshold method (delta-delta $\mathrm{Ct}$ ).

\section{Statistical analyses}

SPSS 17.0 software (SPSS Inc, IBM, Chicago, IL,
USA) was used to perform statistical analyses. Results are expressed as means \pm SEM. Data obtained from T2DM + LIR, T2DM-C and NC groups were examined by one-way ANOVA. Data handing and drawing were processed using GraphPad Prism (v6.0) software. A value of $P<0.05$ was considered statistically significant.

\section{Results}

\section{Body weight and food intake}

T2DM-C and T2DM + LIR rats had similar BWs and food intake before liraglutide treatment. During the treatment period, T2DM + LIR rats showed gradually reduced BW gain (Fig. 1a) and then a significant mean BW loss ( $332 \pm 7$ to $309 \pm 6 \mathrm{~g})$ at the end of 4 weeks. In contrast, the T2DM-C group exhibited the highest mean levels of BW gain (Fig. 1b). Compared with the T2DM + LIR group, food intake was greater in the T2DM-C group, while the NC group showed the lowest food intake (Fig. 1c).

\section{Blood assays}

T2DM-C rats had the highest levels of BG, TG, TC, and FINS (Table 2). However, BG, TC, TG, and FINS were greater in the T2DM + LIR group than the NC group (Table 2).

\section{Immunohistochemistry}

Immunoreactivity for nesfatin-1 was observed in taste bud cells of rat CV. The nesfatin-1-immunoreactive cells were spindle-shaped, with large round nuclei. Immunoreactivity was confined to the cytosol (Fig. 2). We used $\alpha$-gustducin antibody as a type 2 taste cells marker and further investigated that nesfatin-1 and $\alpha$-gustducin were co-expressed in the type 2 taste cells by using adjacent slices (Fig.3). The numbers of nesfatin-1 immunoreactive taste bud cells in each bud of the $\mathrm{CV}$ in T2DM-C rats were significantly less than those in the NC group $(2.32 \pm 0.13$ vs. $3.37 \pm 0.16, P<0.01)$. Interestingly, T2DM + LIR rats showed the most nesfatin-1 positive cells among three groups (T2DM + LIR vs. NC: $4.26 \pm 0.26$ vs. $3.37 \pm 0.16, P<0.01$ ) (Fig. 2).

Table 1 Sequences and efficiency of primers employed for RT-qPCR amplifications

\begin{tabular}{lclll}
\hline Gene & Genebank accession no. & \multicolumn{1}{c}{ Forward primer (5'-3') } & \multicolumn{1}{c}{ Reverse primer (5'-3') } & Product length (bp) \\
\hline NUCB2 & NM_021663.2 & TTGAACACCTGAACCACCAG & AGTCCGGTCATACTGCTCCA & 101 bp \\
Ghrelin & AB029433 & TGGTGTCTTCAGCGACTATCT & CTGCCTCTTCTGCTTGTCCTCT & 204 bp \\
B-Actin & NM_031144.3 & GGAGATTACTGCCCTGGCTCCTA & GACTCATCGTACTCCTGCTTGCTG & 150 bp \\
\hline
\end{tabular}



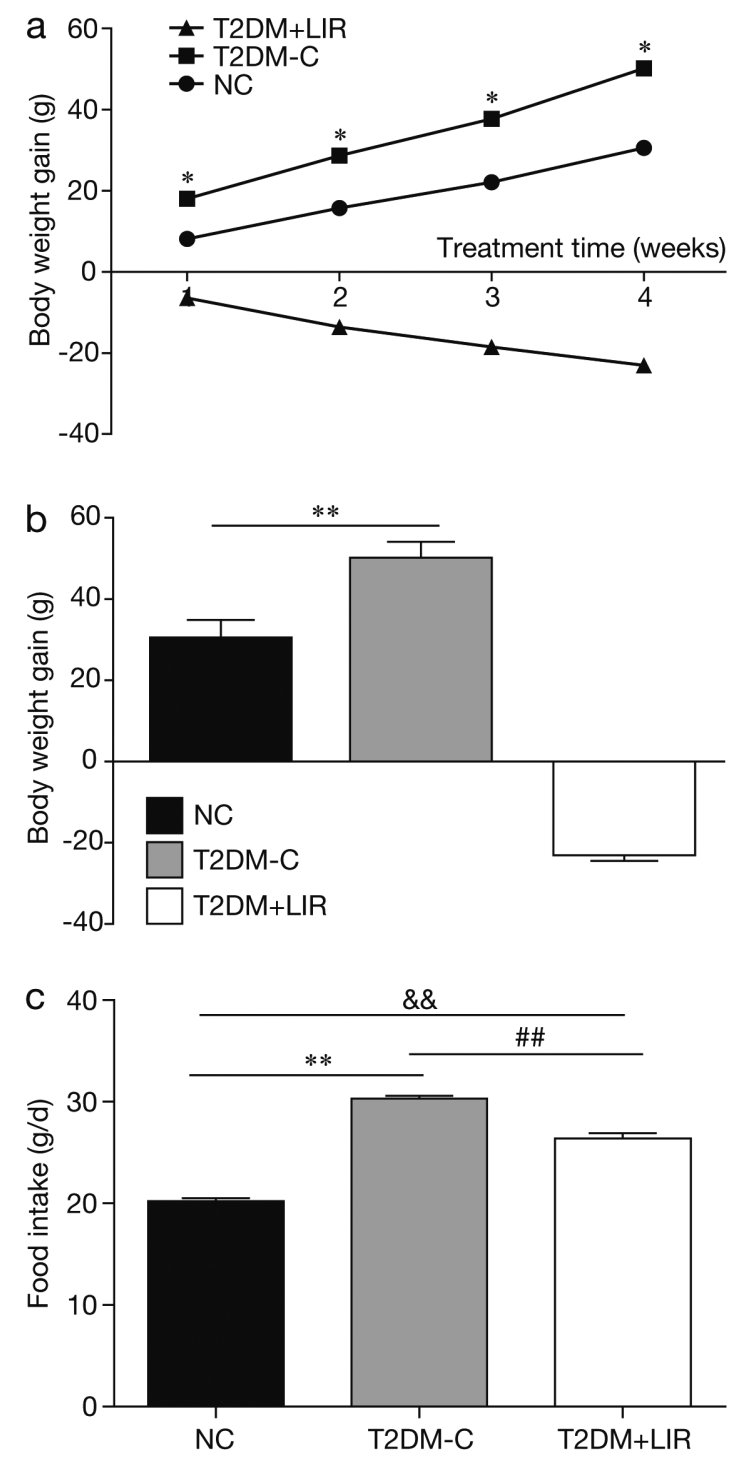

Fig. 1 Body weight gain and food intake in NC, T2DM-C and T2DM+LIR rats. a. Body weight gain of every week during treatment time. b. Total body weight gain after treatment. c. Mean daily food intake during treatment time. *Compared with NC group, $P<0.05, * *$ compared with NC group, $P<0.001$, ${ }^{\# \#}$ compared with T2DM-C group, $P<0.001$, \&\& compared with NC group, $P<0.001$. $\mathrm{NC}$, normal control rats; T2DM-C, diabetic control rats; $\mathrm{T} 2 \mathrm{DM}+\mathrm{LIR}$, diabetic rats treated with liraglutide.

\section{$R T-q P C R$}

RT-qPCR demonstrated that NUCB2 mRNA was highly expressed in the taste buds of rat $\mathrm{CV}$. The relative expression of NUCB2 mRNA in taste buds in rat $\mathrm{CV}(11.32 \pm 0.19)$ was at least 10 -fold higher than in rat hypothalamus (Fig. 4) $(P<0.001)$.

In taste buds, the mean level of NUCB2 mRNA expression was significantly decreased in the T2DM-C group when compared with the NC group (Fig. 5) $(P<0.001)$. T2DM + LIR rats showed the highest expression of NUCB2 mRNA (Fig. 5) $(P<0.001)$. Compared with both the T2DM-C and T2DM + LIR groups, the mean level of ghrelin mRNA in the NC group was significantly reduced (Fig. 5) $(P<0.001)$. However, there was no significant difference in ghrelin mRNA expression between the T2DM-C and T2DM + LIR groups (Fig. 5) $(P>0.05)$.

In the hypothalamus, the mean expression of NUCB2 mRNA in the T2DM-C group was significantly increased compared to that in the $\mathrm{NC}$ or T2DM + LIR groups (Fig. 6) $(P<0.001)$. The lowest level of NUCB2 expression was seen in the NC group (Fig. 6) $(P<0.001)$. Compared with the NC group, the mean expression of ghrelin mRNA in the T2DM-C or T2DM + LIR groups was elevated (Fig. 6) $(P<0.001)$. It was unclear why the changes of mRNA expression of ghrelin in the hypothalamus became more obvious after liraglutide treatment compared to that in the T2DM-C group (Fig. 6) $(P<0.001)$.

\section{Discussion}

There is a strong link between metabolic control and gustation. Taste perception plays a vital sensory role in determining what foodstuffs to ingest and in maintaining metabolic homeostasis. Nesfatin-1 is a gastrointestinal tract hormone classically considered to be associated with appetite control, glucose homeostasis, and slimness. Recent reports have indicated that many peptides in the gastrointestinal tract that regulate appe-

Table 2 BG, TG, TC and FINS levels of NC, T2DM-C and T2DM+LIR rats

\begin{tabular}{|c|c|c|c|c|}
\hline & $\mathrm{BG}(\mathrm{mmol} / \mathrm{L})$ & $\mathrm{TG}(\mathrm{mmol} / \mathrm{L})$ & $\mathrm{TC}(\mathrm{mmol} / \mathrm{L})$ & FINS (ng/mL) \\
\hline $\mathrm{NC}(\mathrm{n}=12)$ & $5.2 \pm 0.1$ & $0.82 \pm 0.01$ & $1.61 \pm 0.01$ & $1.12 \pm 0.01$ \\
\hline T2DM-C $(n=12)$ & $18.9 \pm 0.4^{* * \# \#}$ & $1.78 \pm 0.03^{* * \# \#}$ & $2.57 \pm 0.03^{* * \# \#}$ & $1.67 \pm 0.02^{* * \# \#}$ \\
\hline $\mathrm{T} 2 \mathrm{DM}+\mathrm{LIR}(\mathrm{n}=12)$ & $8.4 \pm 0.4^{\$ \$}$ & $1.29 \pm 0.03^{\$ \$}$ & $2.32 \pm 0.04^{\$ \$}$ & $1.24 \pm 0.01^{\$ \$}$ \\
\hline
\end{tabular}

NC, normal control rats; T2DM-C, diabetic control rats; T2DM+LIR, diabetic rats treated with liraglutide. BG, blood glucose; TG,

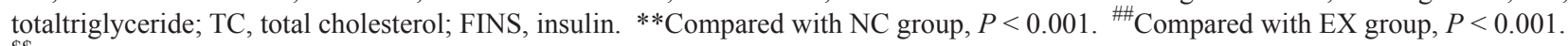
$\$ \$$ Compared with NC group, $P<0.001$. 

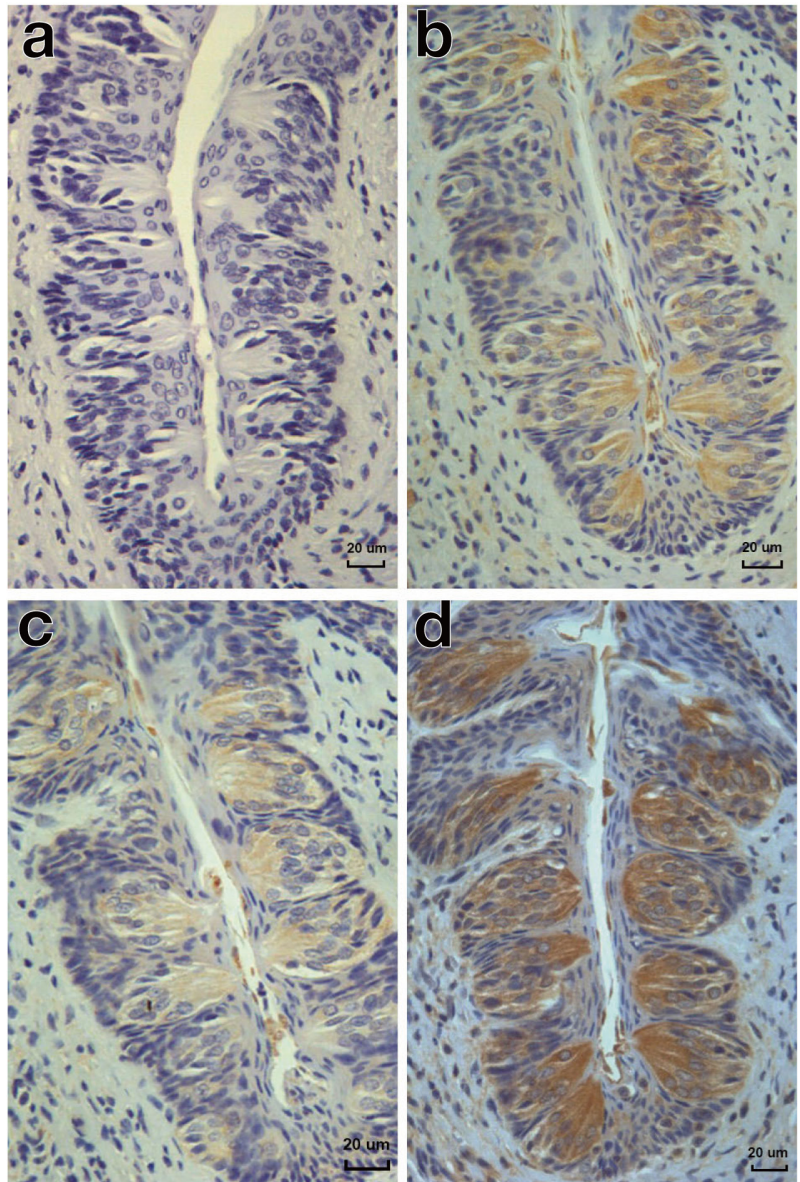

Fig. 2 Nesfatin-1 immunolocalization in circumvallate taste buds of NC, T2DM-C and T2DM+LIR rats. a. Negative control section where the primary antibody was omitted. b. Nesfatin-1 immunopositive cells are spindle-shaped and contain intensely labeled cytoplasm in circumvallate papillae of $\mathrm{NC}$ rats. c. Nesfatin-1 immunopositive taste cells were less expressed in T2DM-C rats than NC rats. d. T2DM+LIR rats showed the most Nesfatin-1 positive cells among three groups. Scale bar $=20 \mu \mathrm{m}$.

tite are co-expressed in taste bud cells [9-12], where they may play a modulatory role in fine tuning taste perception. Therefore, it is reasonable to speculate that nesfatin- 1 is expressed in taste bud cells. In this study, we successfully verified the expression of nesfatin- 1 in the type 2 taste buds of rat CV by immunohistochemical staining. It is reported that type 2 taste cells transduce sweet, bitter, or umami stimuli [29], and utilize a $\mathrm{G}$ protein-coupled transduction cascade for signaling $[30,31]$. This result provides insights into the distinct functions of NUCB2/nesfatin-1 within the gustatory system, and suggests it has the potential to influence the amounts and types of food ingested.

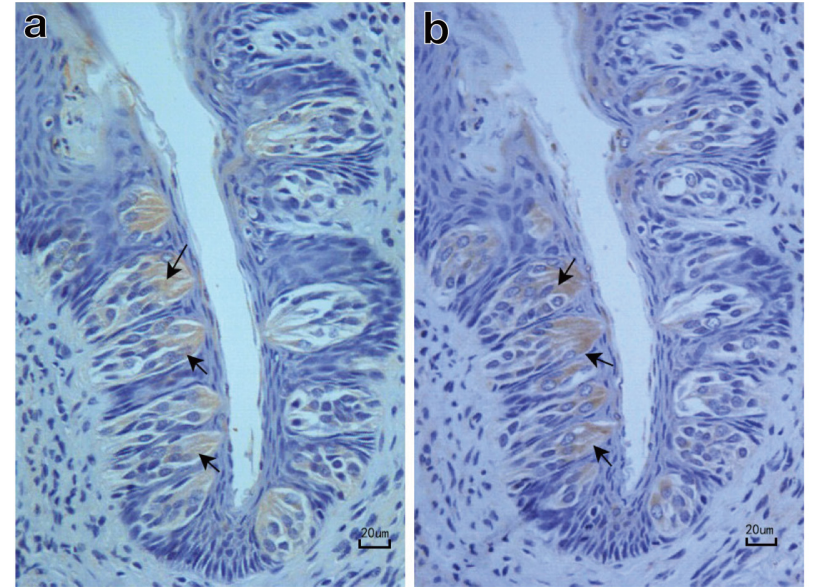

Fig. 3 Co-expression of nesfatin-1 and $\alpha$-gustducin in the type 2 taste cells of rat circumvallate papillae (CV) by using adjacent slices. a. Nesfatin-1 is expressed in the taste cells of rat CV. b. $\alpha$-gustducin is expressed in the taste cells of rat CV. Arrows, cell expressing both nesfatin-1 and $\alpha$-gustducin. Scale bar $=20 \mu \mathrm{m}$.

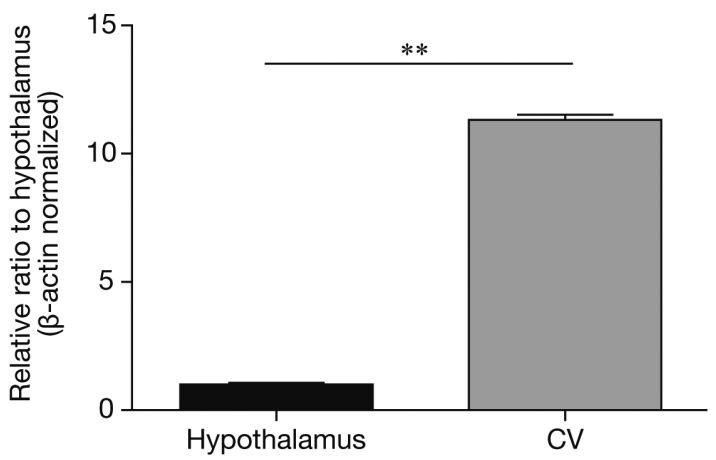

Fig. 4 NUCB2 mRNA expression in the CV and hypothalamus of rats. The precursor of nesfatin-1, NUCB2, was significantly more highly expressed in the CV than in the hypothalamus. Data are expressed as mean \pm sem. $* *, P$ $<0.001 \mathrm{vs}$. hypothalamus. CV, circumvallate papillae.

Patients with T2DM often present with clinical symptoms of polyphagia, polydipsia, and polyuria. Chronic hyperglycemia in T2DM also can induce chronic complications that can affect almost every organ system of the body. Taste impairment is one such complication of T2DM [32]. However, the exact pathogenesis of taste impairment in diabetes is unclear.

Liraglutide, a classical GLP-1 analog, is used to treat T2DM. There is increasing evidence that liraglutide can reduce food intake, BW, and BG [21]. However, there is little known about the exact mechanism of GLP-1R agonists, especially in taste perception. In the current study, we successfully showed that: 

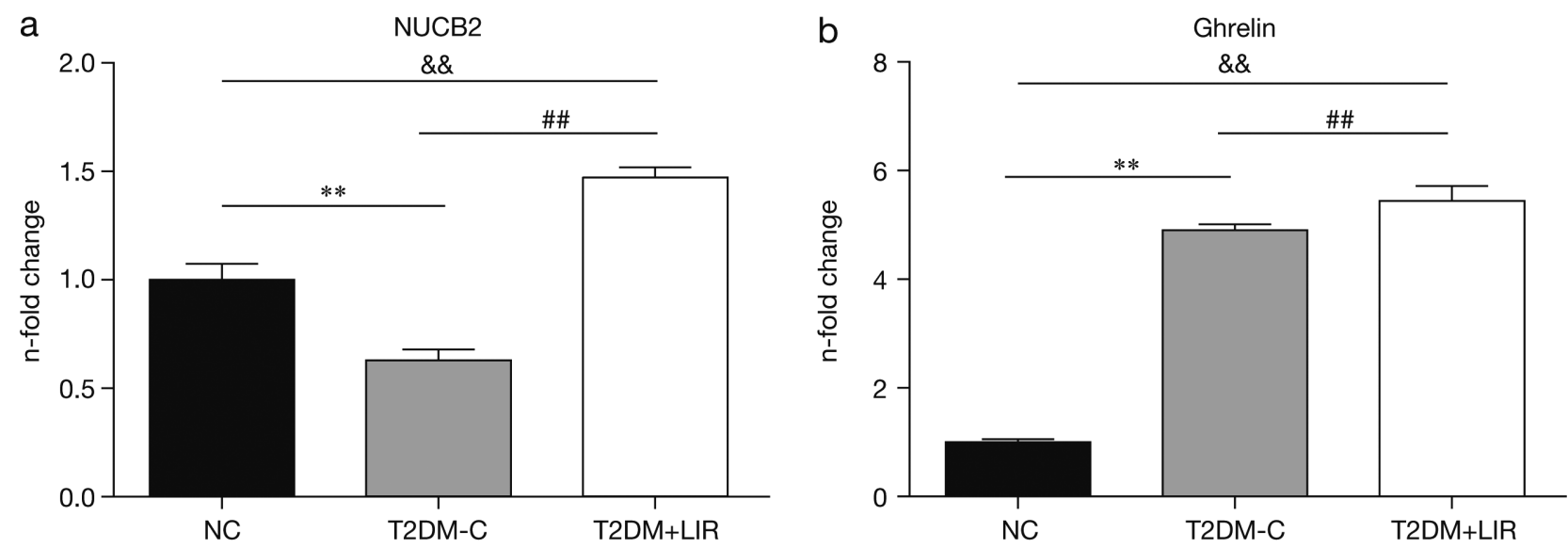

Fig. 5 mRNA expressions of NUCB2 and ghrelin in the CV of NC, T2DM-C and T2DM+LIR rats. a. NUCB2. b. Ghrelin. **Compared with NC group, $P<0.001$, \#\# compared with T2DM-C group, $P<0.001$, \& \& compared with NC group, $P<0.001$. NC, normal control rats; T2DM-C, diabetic control rats; T2DM+LIR, diabetic rats treated with liraglutide. NUCB2, the precursor of nesfatin-1.
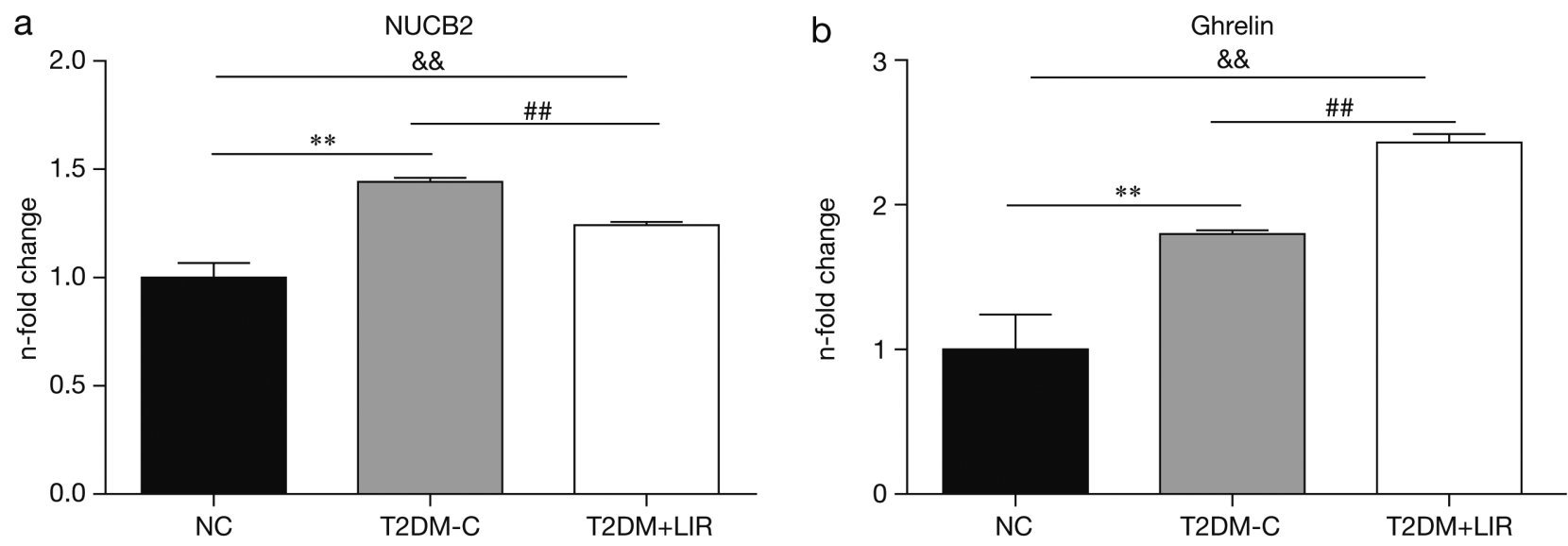

Fig. 6 mRNA expressions of NUCB2 and ghrelin in the hypothalamus of NC,T2DM-C and T2DM+LIR rats. a. NUCB2. b. Ghrelin. ${ }^{* *}$ Compared with NC group, $P<0.001,{ }^{\# \#}$ compared with T2DM-C group, $P<0.001$, \&\& compared with NC group, $P<0.001$. $\mathrm{NC}$, normal control rats; T2DM-C, diabetic control rats; T2DM+LIR, diabetic rats treated with liraglutide. NUCB2, the precursor of nesfatin-1.

1) NUCB2 mRNA is expressed in rat $\mathrm{CV}, 2$ ) the expression level of NUCB2 mRNA is decreased in taste buds of T2DM-C rats, and 3) liraglutide treatment can significantly elevate the expression of NUCB2 mRNA in the $\mathrm{CV}$ of diabetic rats. These findings are beneficial to further understand the effects and interactions of nesfatin-1 and GLP-1 on the regulation of taste sensitivity, feeding, and energy balance, as well as the pathogenesis of obesity and/or diabetes.

The results of the current study showed that the expression of NUCB2 mRNA in the hypothalamus of T2DM-C rats was increased. This result is consistent with most previous studies [33] and suggested that
NUCB2 secretion in the hypothalamus may be a compensatory mechanism in early stages of T2DM by suppressing food intake and reducing BW to improve insulin resistance and lipid metabolic disorders. However, in the hypothalamus, NUCB2 expression was decreased in T2DM + LIR compared to T2DM-C rats. These data suggest that in same situation, the expression of NUCB2 in taste buds and the hypothalamus may differ. However, the functional nature of these phenomena in terms of local or whole body biology remains to be explored.

In T2DM-C rats, the reduced expression of NUCB2 in $\mathrm{CV}$ taste buds may promote excess food intake and 
BW gain, while the elevation of NUCB2 expression in the hypothalamus may be an indirect compensation for decreased NUCB2 in the CV. We observed a decreased $\mathrm{BW}$ gain, food intake, and $\mathrm{BG}$ in T2DM + LIR rats. The increased NUCB2 in the CV induced by liraglutide might be involved in this effect. However, in the hypothalamus, the decreased expression of NUCB2 in T2DM + LIR rats should be a negative effect for weight loss. Nevertheless, the result was beneficial, although the mechanism remains unclear.

Interestingly, whether in taste buds or in hypothalamus, the expression of NUCB2 in T2DM + LIR group was actually higher than that of normal rats. A recent study by Katsurada et al. [34] indicated that endogenous GLP-1 targets the paraventricular nucleus to restrict feeding behavior. This implicates the projection from the nucleus tractus solitarius GLP-1 neurons, activation of corticotropin-releasing hormone and nesfatin-1 neurons. Therefore, we think that liraglutide probably has a direct effect on the expression of nucleobindin-2 independent of the glycemic states.

Ghrelin opposes nesfatin-1 in the regulation of energy balance. In the current study, we found that, throughout the hypothalamus and CV, the mean level of ghrelin in either T2DM-C or T2DM + LIR rats was significant higher than in the NC group. Ghrelin levels were highest in T2DM + LIR rats, and never less than the T2DM-C group in the CV. The elevated expression of ghrelin in the hypothalamus of T2DM-C rats may be associated with increased caloric intake during the course of obesity-related T2DM. However, it is surprising that the mean expression level of ghrelin in T2DM + LIR rats was higher than that in the T2DM-C group. This finding indicates that, in the CV, ghrelin expression is independent of the GLP-1R pathway, while in the hypothalamus ghrelin secretion may be a compensatory response to liraglutide treatment.

The results of the current study firmly establish that nesfatin-1 and ghrelin are co-expressed in the taste buds of rat $\mathrm{CV}$. Interestingly, the ghrelin level is elevated, while the NUCB2 level is decreased, in the $\mathrm{CV}$ of T2DM-C rats. This is consistent with the earlier finding that there is a reciprocal relationship between nesfatin-1 and ghrelin in gastrointestinal endocrine cells [7]. Our data further indicate that taste buds of rat $\mathrm{CV}$, like gastric endocrine cells play an important role in regulating food intake. However, the situation appears to be different in the hypothalamus, which is a key integration area of the brain where numerous appe- tite-regulating peptides are released to participate in the control of energy homeostasis. In the arcuate nucleus of the hypothalamus, there are two distinct neuronal populations: one is a group of neurons co-expressing orexigenic neuropeptides, including neuropeptide $\mathrm{Y}$ and agouti-related protein; the other is a subset of neurons expressing anorexigenic neuropeptides, including proopiomelanocortin and cocaine- and amphetamineregulated transcript. These are first-order neurons where peripheral metabolic signals, including peripheral adiposity signals (leptin, insulin, etc.), appetite regulating hormones (CCK, GLP-1, PYY, nesfatin-1, ghrelin, etc.), and nutrients are primarily transferred [35]. Therefore, the expression level of single or even double peptides such as nesfatin-1 or ghrelin in the hypothalamus may not fully reveal the complex network of hypothalamic appetite regulation.

In summary, nesfatin- 1 is expressed in the type 2 taste bud cells of the CV. This indicates that nesfatin-1 may have some effects on the gustatory systems. The decreased expression of NUCB2 in the CV of T2DM-C rats is a possible cause of diabetic taste impairment. Moreover, liraglutide can regulate appetite and energy homeostasis through effects on the gustatory system. The increased expression of NUCB2 in the CV after liraglutide treatment might play a role in this effect. However, the results of the current study could not provide a definitive mechanism of liraglutide actions on the hypothalamus or the whole body. In T2DM rats, nesfatin- 1 and ghrelin may be secreted simultaneously from the $\mathrm{CV}$, but regulated individually to play opposite roles in appetite regulation. This suggests that the gustatory system is involved in the regulation of the body's energy balance. The regulation of appetite involves complex mechanisms, many endocrine organs (hypothalamus, taste buds, gastrointestinal tract, etc.) and many hormone signals (nesfatin-1, ghrelin, GLP1, etc.). Further studies designed to explore the interaction between NUCB2/nesfatin-1 and other appetiteregulatory peptides are needed.

\section{Acknowledgments}

The authors are grateful to all study participants.

\section{Disclosure}

The authors declare that there is no conflict of interest. 


\section{References}

1. Oh-I S, Shimizu H, Satoh T, Okada S, Adachi S, et al. (2006) Identification of nesfatin-1 as a satiety molecule in the hypothalamus. Nature 443: 709-712.

2. Stengel A, Goebel M, Wang L, Rivier J, Kobelt P, et al. (2009) Central nesfatin-1 reduces dark-phase foodintake and gastric emptying in rats: differential role of corticotropin-releasing factor2 receptor. Endocrinology 150:4911-4919.

3. Nakata M, Manaka K, Yamamoto S, Mori M, Yada $\mathrm{T}$ (2011) Nesfatin-1 enhances glucose-induced insulin secretion by promoting $\mathrm{Ca} 2+$ influx through L-type channels in mouse islet beta-cells. Endocr J 58: 305-313.

4. Gonzalez R, Perry RL, Gao X, Gaidhu MP, Tsushima $R G$, et al. (2011) Nutrient responsive nesfatin-1 regulates energy balance and induces glucose-stimulated insulin secretion in rats. Endocrinology 152: 3628-3637.

5. Pan W, Hsuchou H, Kastin AJ (2007) Nesfatin-1 crosses the blood-brain barrier without saturation. Peptides 28: 2223-2228.

6. Shimizu H, Inoue K, Mori M (2007) The leptin-dependent and-independent melanocortin signaling system: regulation of feeding and energy expenditure. $J$ Endocrinol 193: 1-9.

7. Stengel A, Goebel M, Yakubov I, Wang L, Witcher D, et al. (2009) Identification and characterization of nesfatin-1 immunoreactivity in endocrine cell types of the rat gastric oxyntic mucosa. Endocrinology 150: 232-238.

8. Zhang AQ, Li XL, Jiang CY, Lin L, Shi RH, et al. (2010) Expression of nesfatin-1/NUCB2 in rodent digestive system. World J Gastroenterol 16: 1735-1741.

9. Herness S, Zhao FL, Lu SG, Kaya N, Shen T (2002) Expression and physiological actions of cholecystokinin in rat taste receptor cells. J Neurosci 22: 10018-10029.

10. Shin YK, Martin B, Golden E, Dotson CD, Maudsley S, et al. (2008) Modulation of taste sensitivity by GLP-1 signaling. J Neurochem 106: 455-463.

11. Martin B, Dotson CD, Shin YK, Ji S, Drucker DJ, et al. (2009) Modulation of Taste Sensitivity by GLP-1 Signaling in Taste Buds. Ann NY Acad Sci 1170: 98-101.

12. Shin YK, Martin B, Kim W, White CM, Ji S, et al. (2010) Ghrelin is produced in taste cells and ghrelin receptor null mice show reduced taste responsivity to salty $(\mathrm{NaCl})$ and sour (citric acid) tastants. PLoS One 5:811-829.

13. Kojima M, Hosoda H, Date Y, Nakazato M, Matsuo $\mathrm{H}$, et al. (1999) Ghrelin is a growth-hormone-releasing acylated peptide from stomach. Nature 402: 656-660.

14. Mojsov S, Weir GC, Habener JF (1987) Insulinotropin: glucagon-like peptide I (7-37) co-encoded in the glucagon gene is a potent stimulator of insulin release in the perfused rat pancreas. J Clin Invest 79: 616-619.

15. Kreymann B, Williams G, Ghatei MA, Bloom SR (1987) Glucagon-like peptide-1 7-36: a physiological incretin in man. Lancet 330: 1300-1304.

16. Dailey MJ, Moran TH (2013) Glucagon-like peptide 1 and appetite. Trends Endocrinol Metab 24: 85-91.

17. Knop FK, Vilsboll T, Larsen S, Madsbad S, Holst JJ, et al. (2003) No hypoglycemia after subcutaneous administration of glucagon-like peptide- 1 in lean type 2 diabetic patients and in patients with diabetes secondary to chronic pancreatitis. Diabetes Care 26: 2581-2587.

18. Le Floch JP, Le Lievre G, Sadoun J, Perlemuter L, Peynegre R, et al. (1989) Taste impairment and related factors in type I diabetes mellitus. Diabetes Care 12: 173-178.

19. Hardy SL, Brennand CP, Wyse BW (1981) Taste thresholds of individuals with diabetes mellitus and of control subjects. J Am Diet Assoc 79: 286-289.

20. Stolbová K, Hahn A, Benes B, Andel M, Treslová L (1998) Gustometry of diabetes mellitus patients and obese patients. Int Tinnitus J 5: 135-140.

21. Degn KB, Juhl CB, Sturis J, Jakobsen G, Brock B, et al. (2004) One week's treatment with the long-acting glucagon-like peptide 1 derivative liraglutide (NN2211) markedly improves 24-h glycemia and $\alpha$ - and $\beta$-cell function and reduces endogenous glucose release in patients with type 2 diabetes. Diabetes 53: 1187-1194.

22. Feng XH, Liu XM, Zhou LH, Wang J, Liu GD (2008) Expression of glucagon-like peptide-1 in the taste buds of rat circumvallate papillae. Acta Histochem 110: 151-154.

23. Feng XH, Zhou LH, Wang D, Yuan X (2012) GLP-1 expression in von Ebner's gland of diabetic rats. Peptides 38: 137-141.

24. Zhang XJ, Wang YQ, Long Y, Wang L, Li Y, et al. (2013) Alteration of sweet taste in high-fat diet induced obese rats after 4weeks treatment with exenatide. Peptides 47: 115-123.

25. Ramesh N, Mortazavi S, Unniappan S (2015) Nesfatin-1 stimulates glucagon-like peptide-1 and glucose-dependent insulinotropic polypeptide secretion from STC-1 cells in vitro. Biochem Biophys Res Commun 462:124130.

26. Srinivasan K, Viswanad B, Asrat L, Kaul CL, Ramarao $\mathrm{P}$ (2005) Combination of high-fat diet-fed and low-dose streptozotocin-treated rat: a model for type 2 diabetes and pharmacological screening. Pharmacol Res 52: 313-320.

27. Shi SR, Cote RJ, Liu C, Yu MC, Castelao JE, et al. (2002) A modified reduced-temperature antigen retrieval protocol effective for use with a polyclonal antibody to cyclooxygenase-2 (PG 27). Appl Immunohistochem Mol Morphol 10:368-373.

28. Shen T, Kaya N, Zhao FL, Lu SG, Cao Y, et al. (2005) Co-expression patterns of the neuropeptides vasoactive intestinal peptide and cholecystokin in with the trans- 
duction molecules a-gustducin and T1R2 in rat taste receptor cells. Neuroscience 130: 229-238.

29. Tomchik SM, Berg S, Kim JW, Chaudhari N, Roper SD (2007) Breadth of tuning and taste coding in mammalian taste buds. J Neurosci 27:10840-10848.

30. Chandrashekar J, Hoon MA, Ryba NJ, Zuker CS (2006) The receptors and cells for mammalian taste. Nature 444:288-294.

31. Roper SD (2006) Cell communication in taste buds. Cell Mol Life Sci 63:1494-1500.

32. Ziółkowska J (2006) Oral health status and dental service needs of diabetic patients. Ann Acad Med Stetin 52: 103-114. (In Polish)
33. Zhang Z, Li L, Yang M, Liu H, Boden G, et al. (2011) Increased plasma levels of nesfatin-1 in patients with newly diagnosed type 2 diabetes mellitus. Exp Clin Endocrinol Diabetes 120: 91-95.

34. Katsurada K, Maejima Y, Nakata M, Kodaira M, Suyama S, et al. (2014) Endogenous GLP-1 acts on paraventricular nucleus to suppress feeding: Projection from nucleus tractus solitarius and activation of corticotropin-releasing hormone, nesfatin-1 and oxytocin neurons. Biochem Biophys Res Commun 451: 276-281.

35. Yu JH, Kim MS (2012) Molecular mechanisms of appetite regulation. Diabetes Metab J 36: 391-398. 